\title{
A Novel Class of Iterative Approximation Methods for DSL Spectrum Optimization
}

\author{
Paschalis Tsiaflakis and François Glineur \\ Center for Operations Research and Econometrics, Université catholique de Louvain, B-1348 Louvain-la-Neuve, Belgium \\ Information and Communication Technologies, Electronics and Applied Mathematics Institute, Université catholique de Louvain, \\ B-1348 Louvain-la-Neuve, Belgium; e-mail:paschalis.tsiaflakis@esat.kuleuven.be, francois.glineur@uclouvain.be
}

\begin{abstract}
Spectrum optimization is a promising means to tackle the crosstalk problem in DSL systems, and corresponds to a challenging nonconvex optimization problem. Iterative convex approximation (ICA) methods have been proposed in the literature to deal with this optimization problem. These methods consist in solving a series of improving convex approximations and are typically implemented in a per-user iterative approach. In this paper we develop a novel class of iterative methods that focus explicitly on per-user iterative implementations, and which consist of improved per-user approximations that are tighter and simpler to solve (in closed-form) than state-of-theart ICA methods. As a result, the proposed methods improve the convergence speed as fewer approximations are required to converge, and display a significantly lower computational cost. Furthermore, three of the proposed methods can tackle the issue of getting stuck in bad locally optimal solutions, and hence improve solution quality with respect to existing ICA methods.
\end{abstract}

\section{INTRODUCTION}

Modern digital subscriber line (DSL) systems suffer from crosstalk, i.e., electromagnetic interference among different copper lines in the same cable bundle. The presence of crosstalk transforms the DSL access network into a challenging multi-user multi-carrier interference environment, in which the transmission of one user can significantly impact the transmission of all other users.

Dynamic spectrum management (DSM) refers to a promising set of multi-user techniques to tackle this crosstalk problem. In this paper, we will focus on DSM through spectrum optimization, which consists of jointly optimizing the transmit spectra of the interfering users, so as to prevent the impact of crosstalk and to maximize data rates.

The DSM spectrum optimization problem corresponds to a challenging nonconvex problem, for which many DSM algorithms have been proposed in the literature, ranging from fully autonomous [1], [2], [3], and distributed [2], [4], [5], [6], to centralized algorithms [7], [8]. In particular, the approach of iterative convex approximation (ICA) has been recognized to be very efficient, such as the CA-DSB [2] and SCALE [5] algorithms. These algorithms consist in solving a series of improving convex approximations to the nonconvex problem until convergence to a locally optimal solution or a stationary point. The complexity of this ICA approach depends on two factors: (i) the type of approximation, where a tighter approximation generally results in fewer iterations until convergence,

This text presents results of the Belgian Program on Interuniversity Poles of Attraction initiated by the Belgian State, Prime Minister's Office, Science Policy Programming. The scientific responsibility rests with its authors. and (ii) the computational cost of solving the corresponding approximation. Such ICA algorithms (for distributed or centralized computation) are typically implemented in a per-user iterative approach, as this is demonstrated to be highly efficient [3], [2], [9], [4], [5], [6]. For instance, it is shown that these iterative implementations can solve small- to medium-scale DSL scenarios, i.e. up to 6 users, within a few seconds [2]. However, for large-scale DSL scenarios, i.e. 10 to 50 users, these iterative methods can take several minutes. Moreover, it is shown in [2] that these methods can sometimes get stuck in bad locally optimal solutions.

In this paper we design a novel class of iterative approximation methods that explicitly focus on the typical per-user iterative implementation. The proposed methods consist of improved per-user approximations that are much tighter and simpler to solve than existing state-of-the-art ICA methods. More specifically, we introduce IASB1 and IASB2, which consist of improved per-user convex approximations, as well as IASB3, IASB4 and IASB5, which consist of per-user nonconvex approximations. We will show that the corresponding per-user approximations can easily be solved in closed-form, i.e., by computing the roots of a linear, quadratic or cubic polynomial, even for large-scale DSL scenarios, in contrast to existing ICA methods. Furthermore, we will show how IASB3, IASB4 and IASB5 can tackle the issue of getting stuck in bad locally optimal solutions. Both a reduction in the number of iterations required for convergence and an improvement in solution quality (i.e., improved data rate performance) will be demonstrated using a realistic DSL simulator.

\section{System Model and Spectrum Optimization}

We consider a cable bundle that consists of a set $\mathcal{N}=$ $\{1, \ldots, N\}$ of $N$ DSL users (i.e., lines) with the standard assumption of synchronous discrete multi-tone (DMT) modulation and with a set $\mathcal{K}=\{1, \ldots, K\}$ of $K$ tones (i.e., frequency carriers). We assume no signal coordination at the transmitters and at the receivers. Furthermore, we assume that the interference is treated as additive white Gaussian noise. Under these standard assumptions for DSL spectrum coordination, the spectrum optimization problem (SO problem) can be formulated as follows

$$
\begin{array}{ll}
\text { (SO problem): } & \min _{\mathbf{s}_{k} \in \mathcal{S}_{k}, k \in \mathcal{K}} \sum_{k \in \mathcal{K}} f_{k}\left(\mathbf{s}_{k}\right) \\
& \text { subject to } \quad \sum_{k \in \mathcal{K}} \mathbf{s}_{k} \leq \mathbf{P}^{\text {tot }},
\end{array}
$$


with $\mathbf{s}_{k}=\left[s_{k}^{1}, \ldots, s_{k}^{N}\right]^{T}$ denoting transmit powers of all users on tone $k$, the vector constant $\mathbf{P}^{\text {tot }}=\left[P^{1, \text { tot }}, \ldots, P^{N \text {,tot }}\right]^{T}$ denoting the total power budgets for all users, and the set $\mathcal{S}_{k}=\left\{s_{k}^{n}, n \in \mathcal{N} \mid 0 \leq s_{k}^{n} \leq s_{k}^{n \text {,mask }}, \forall n \in \mathcal{N}\right\}$ denoting the feasible set on each tone $k$ with constant spectral mask $s_{k}^{n \text {,mask }}$ for user $n$. Each term $f_{k}\left(\mathbf{s}_{k}\right)$ in the objective function corresponds to the following nonconvex function

$$
f_{k}=f_{k}\left(\mathbf{s}_{k}\right)=-\sum_{n \in \mathcal{N}} w_{n} \log \left(1+\frac{s_{k}^{n}}{\sum_{m \neq n} a_{k}^{n, m} s_{k}^{m}+z_{k}^{n}}\right),
$$

with $w_{n}$ denoting the weight given to the data rate of user $n$, $a_{k}^{n, m}$ denoting the normalized channel gains from transmitter $m$ to receiver $n$ on tone $k$, and $z_{k}^{n}$ denoting the normalized noise power for line $n$ on tone $k$. The standard SNR gap is also included in the normalized channel gains and noise power.

\section{Spectrum Optimization Through Iterative CONVEX APPROXIMATION}

The ICA approach to tackle the SO problem consists in solving a series of improving convex approximations. This procedure is given in Algorithm 1.

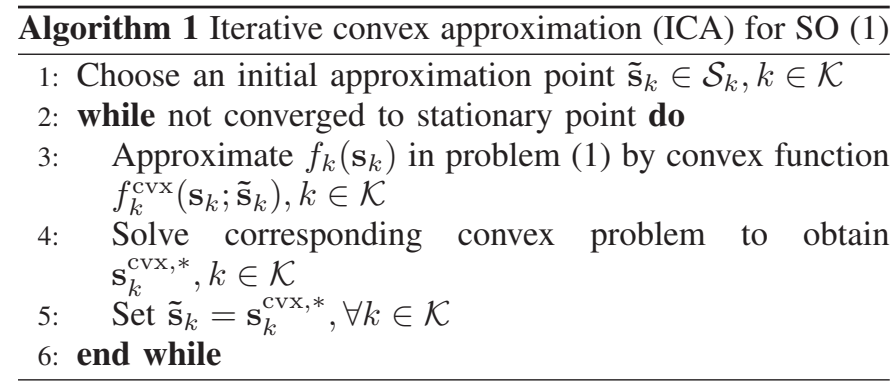

The ICA procedure is guaranteed to converge to a locally optimal solution or a stationary point of the SO problem under the following conditions [10]:

$$
\begin{gathered}
f_{k}^{\operatorname{cvx}}\left(\tilde{\mathbf{s}}_{k} ; \tilde{\mathbf{s}}_{k}\right)=f_{k}\left(\tilde{\mathbf{s}}_{k}\right), \forall k \in \mathcal{K}, \\
\nabla f_{k}^{\operatorname{cvx}}\left(\tilde{\mathbf{s}}_{k} ; \tilde{\mathbf{s}}_{k}\right)=\nabla f_{k}\left(\tilde{\mathbf{s}}_{k}\right), \forall k \in \mathcal{K}, \\
f_{k}^{\operatorname{cvx}}\left(\mathbf{s}_{k} ; \tilde{\mathbf{s}}_{k}\right) \geq f_{k}\left(\mathbf{s}_{k}\right), \forall \mathbf{s}_{k} \in \mathcal{S}_{k}, k \in \mathcal{K},
\end{gathered}
$$

which impose that the approximation $f_{k}^{\mathrm{cvx}}\left(\mathbf{s}_{k} ; \tilde{\mathbf{s}}_{k}\right)$ coincides at first-order with the true objective function $f_{k}\left(\mathbf{s}_{k}\right)$ around the approximation point $\tilde{\mathbf{s}}_{k}, k \in \mathcal{K}$, and also that the approximation is an upper bound on the true objective function. The CADSB and SCALE algorithms are two effective ICA procedures that, respectively, use the convex approximations $f_{k}^{\mathrm{CADSB}}$ and $f_{k}^{\text {SCALE }}$ of Table I in which parameters $b_{k}^{n}\left(\tilde{\mathbf{s}}_{k}\right), \alpha_{k}^{n}\left(\tilde{\mathbf{s}}_{\mathbf{k}}\right)$ and $c_{k}^{n}\left(s_{k}^{-n}, \tilde{\mathbf{s}}_{k}\right)$ are constants that depend on the approximation point $\tilde{s}_{k}$, and which are computed in closed-form such that conditions (3-5) are satisfied, see [2], [5].

For the concrete implementation of ICA, one typically follows an iterative approach (for centralized [2], [9] as well as distributed implementations [1], [2], [4], [5], [6]), i.e., each user iteratively computes its own transmit powers taking the fixed interference of the other users into account. This comes down to the typical per-user iterative implementation as shown in Algorithm 2, in which $s_{k}^{-n}$ refers to the vector containing

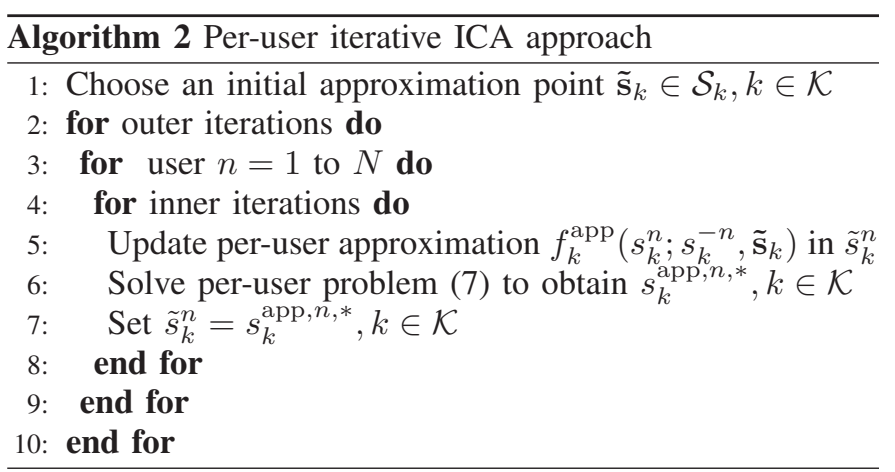

transmit powers on tone $k$ of all users except for user $n$, and lines 4 to 8 focus on solving the following per-user version of the SO problem:

$$
\begin{array}{ll}
\min _{s_{k}^{n} \in \mathcal{S}_{k}^{n}, k \in \mathcal{K}} & \sum_{k \in \mathcal{K}} f_{k}\left(s_{k}^{n} ; s_{k}^{-n}\right) \\
\text { subject to } & \sum_{k \in \mathcal{K}} s_{k}^{n} \leq \mathbf{P}^{n, \text { tot }},
\end{array}
$$

with $\mathcal{S}_{k}^{n}=\left\{s_{k}^{n} \mid 0 \leq s_{k}^{n} \leq s_{k}^{n \text {,mask }}\right\}$. More specifically, lines 4 to 8 come down to solving (6) with an iterative approximation approach, where each approximation (line 6 of Algorithm 2) corresponds to the following per-user problem

$$
\begin{array}{ll}
\min _{s_{k}^{n} \in \mathcal{S}_{k}^{n}, k \in \mathcal{K}} & \sum_{k \in \mathcal{K}} f_{k}^{\text {app }}\left(s_{k}^{n} ; s_{k}^{-n}, \tilde{\mathbf{s}}_{k}\right) \\
\text { subject to } & \sum_{k \in \mathcal{K}} s_{k}^{n} \leq \mathbf{P}^{n, \text { tot }} .
\end{array}
$$

Note that we do not require that the approximation function $f_{k}^{\text {app }}$ we use to be convex. This per-user problem is typically solved by focusing on its dual problem formulation as follows:

$$
\begin{gathered}
\max _{\lambda_{n} \geq 0} g\left(\lambda_{n}\right) \quad \text { with } \\
g\left(\lambda_{n}\right):=-\lambda_{n} P^{n, \text { tot }}+\sum_{k \in \mathcal{K}}\left[\min _{s_{k}^{n} \in \mathcal{S}_{k}^{n}} f_{k}^{\text {app }}\left(s_{k}^{n} ; s_{k}^{-n}, \tilde{\mathbf{s}}_{k}\right)+\lambda_{n} s_{k}^{n}\right] .
\end{gathered}
$$

The dual problem (8) is a one-dimensional problem in the dual variable $\lambda_{n}$, which can be solved using a simple bisection search, or (sub-)gradient update approaches [8]. However, the evaluation of the objective function $g\left(\lambda_{n}\right)$ corresponds to an optimization problem on its own, i.e., (9). This problem can be decomposed over tones for a given $\lambda_{n}$, resulting in $K$ independent one-dimensional subproblems, which are convex subproblems if $f_{k}^{\text {app }}\left(s_{k}^{n} ; s_{k}^{-n}, \tilde{\mathbf{s}}_{k}\right)$ is convex in $s_{k}^{n}$. This is also referred to as dual decomposition. It can be shown that for CA$\mathrm{DSB}$, i.e., $f_{k}^{\text {app }}=f_{k}^{\mathrm{CADSB}}$, and SCALE, i.e., $f_{k}^{\text {app }}=f_{k}^{\mathrm{SCALE}}$, these decomposed subproblems in (9) can be solved in closedform by computing the roots of a polynomial of degree $N$, which is only simple to obtain when $N<5$, i.e., for DSL scenarios with less than 5 users. Therefore iterative fixed point updates were proposed in [2], [5] to solve the subproblems in (9), instead of computing closed-form solutions. 
TABLE I

PER-USER OBJECTIVE AND APPROXIMATION FUNCTIONS, AND DEGREE OF CORRESPONDING POLYNOMIAL TO SOLVE SUBPROBLEMS IN (9)

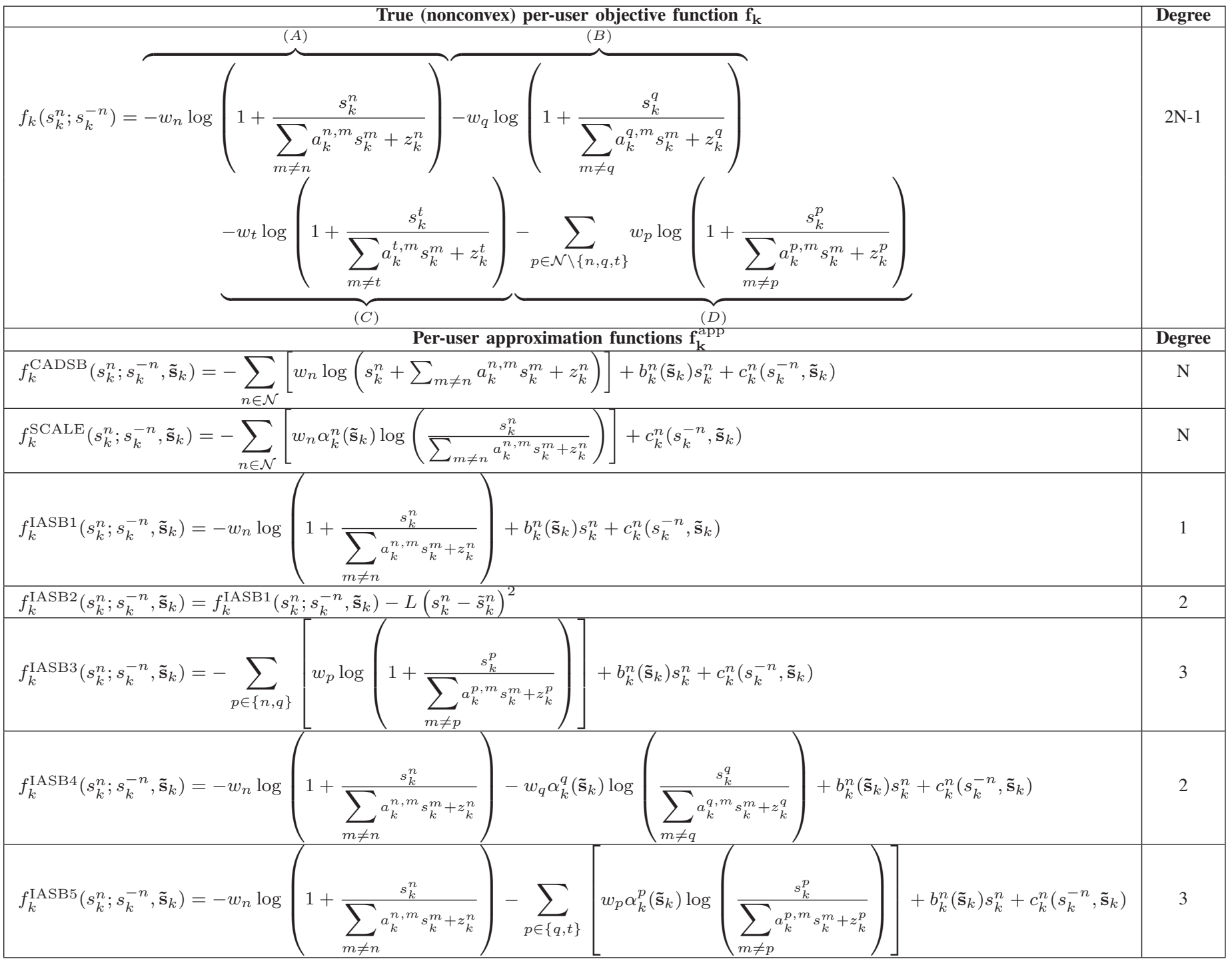

The per-user iterative approach using dual decomposition is recognized as being very effective in tackling the SO problem in the sense that it can find locally optimal solutions to the SO problem with only small computational cost. More specifically, in [2] it is shown that the SO problem up to dimension $K N=$ 2000 can be solved within a few seconds.

However, there are also some drawbacks when using existing ICA methods, such as CA-DSB and SCALE. First, the SO problem can have many locally optimal solutions, depending on the considered DSL scenario, where many of those locally optimal solutions can correspond to a quite suboptimal data rate performance, as demonstrated in [2]. Existing ICA methods feature no mechanism to tackle this issue and, depending on the chosen initial point and the considered DSL scenario, may converge to a locally optimal solution with very seriously deteriorated data rate performance. A second issue is that for large-scale DSL scenarios with 50-100 users and more than 1000 tones, ICA methods may take several minutes or even hours. Any improvement to reduce the execution time is desirable for such large-scale scenarios.

\section{A Novel Class of Per-User Iterative APPROXIMATION METHODS}

In this section, we propose a class of novel per-user iterative methods that rely on five new per-user approximations $f_{k}^{\text {app }}\left(s_{k}^{n} ; s_{k}^{-n}, \tilde{\mathbf{s}} k\right)$ for which the subproblems in (9) can be easily solved in closed-form, i.e., by computing the roots of a linear, quadratic, or cubic polynomial, even for largescale DSL scenarios. This is much simpler than existing ICA methods for which the closed-form solution requires computing the roots of a polynomial of degree $N$, as mentioned in Section III. Next to the significant reduction in computational cost for closed-form solutions, we show that these novel approximations are tighter than those of existing ICA methods, and can even result in improving final solution quality. 


\section{A. Design Approach}

For the design of our new approximations, we start from the true (nonconvex) per-user objective $f_{k}\left(s_{k}^{n} ; s_{k}^{-n}\right)$ as given in Table I, which is a one-dimensional function in $s_{k}^{n}$ that consists of a convex term (A) and concave terms (B), (C) and (D). Then, we replace some of the concave terms by either a linear or a nonlinear first-order approximation around current approximation point $\tilde{\mathbf{s}}_{k}$, so as to obtain a per-user overapproximation $f_{k}^{\text {app }}\left(s_{k}^{n} ; s_{k}^{-n}, \tilde{\mathbf{s}}_{k}\right)$ that satisfies convergence conditions (3-5) in $s_{k}^{n}$. This approximation is then used in Algorithm 2, where (7) is solved using the dual approach (8-9). Our proposed per-user approximations are carefully chosen so that the corresponding KKT stationarity condition, valid for each subproblem in (9),

$$
\frac{\partial}{\partial s_{k}^{n}} f_{k}^{\mathrm{app}}\left(s_{k}^{n} ; s_{k}^{-n}, \tilde{\mathbf{s}}_{k}\right)+\lambda_{n}=0
$$

can be rewritten as a polynomial equation in $s_{k}^{n}$ that can be easily solved. Solution of the subproblems in (9) is then either one of the roots of this polynomial or one of the endpoints of the feasible interval $\mathcal{S}_{k}^{n}$.

\section{B. Novel Methods}

1) Iterative Approximation Spectrum Balancing 1 (IASB1): Our first method corresponds to Algorithm 2 with the peruser approximation function $f_{k}^{\text {app }}=f_{k}^{\text {IASB1 }}\left(s_{k}^{n} ; s_{k}^{-n}, \tilde{\mathbf{s}}_{k}\right)$, as given in Table I, which is obtained by a first-order linear approximation of the concave terms (B), (C) and (D) of $f_{k}$, where constants $b_{k}^{n}\left(\tilde{\mathbf{s}}_{k}\right)$ and $c_{k}^{n}\left(s_{k}^{-n}, \tilde{\mathbf{s}}_{k}\right)$ are chosen (in closedform) for the given approximation point $\tilde{\mathbf{s}}_{k}$ so as to satisfy (3-5). Function $f_{k}^{\mathrm{IASB} 1}\left(s_{k}^{n} ; s_{k}^{-n}, \tilde{\mathbf{s}}_{k}\right)$ corresponds to a onedimensional convex function in $s_{k}^{n}$, and satisfies the following:

Lemma 4.1: Approximation $f_{k}^{\mathrm{IASB} 1}\left(s_{k}^{n} ; s_{k}^{-n}, \tilde{\mathbf{s}}_{k}\right)$ is tighter than approximation $f_{k}^{\mathrm{CADSB}}\left(s_{k}^{n} ; s_{k}^{-n}, \tilde{\mathbf{s}}_{k}\right)$, i.e., $f_{k}^{\text {IASB1 }}\left(s_{k}^{n} ; s_{k}^{-n}, \tilde{\mathbf{s}}_{k}\right) \leq f_{k}^{\operatorname{CADSB}}\left(s_{k}^{n} ; s_{k}^{-n}, \tilde{\mathbf{s}}_{k}\right)$

Proof: The proof is straightforward and consists in showing that the second derivative of $f_{k}^{\mathrm{IASB} 1}\left(s_{k}^{n} ; s_{k}^{-n}, \tilde{\mathbf{s}}_{k}\right)$ in $s_{k}^{n}$ is smaller than that of $f_{k}^{\operatorname{CADSB}}\left(s_{k}^{n} ; s_{k}^{-n}\right)$. Omitted for space reasons.

A tighter approximation results in fewer approximations to converge to a local optimum of (6), i.e., fewer inner iterations are required in Algorithm 2. Furthermore, following a dual decomposition approach to solve the corresponding convex approximation (7) with $f_{k}^{\text {app }}=f_{k}^{\text {IASB1}}$, the decoupled subproblems in (9) can be solved in closed-form as follows

$$
s_{k}^{n}=\left[\frac{w_{n}}{\lambda_{n}+b_{k}^{n}\left(\tilde{\mathbf{s}}_{k}\right)}-\left(\sum_{m \neq n} a_{k}^{n, m} s_{k}^{m}+z_{k}^{n}\right)\right]_{0}^{s_{k}^{n, \text { mask }}}
$$

where $[x]_{a}^{b}$ means $\max (\min (x, b), a)$, and which we obtain by extracting $s_{k}^{n}$ from (10) with $f_{k}^{\text {app }}=f_{k}^{\text {IASB1 }}$, and projecting on the feasible interval $\mathcal{S}_{k}^{n}$. The proposed per-user approximation is thus both tighter than that of CA-DSB, and much easier to solve (in closed-form) than CA-DSB and SCALE. The computational costs are summarized in the last column of Table I. A concrete improvement in number of approximations and complexity will be demonstrated in Section VI.

Finally we want to note that the update formula (11) of IASB 1 corresponds to that of the DSB algorithm proposed in
[2]. However, both update formulas are derived in a fundamentally different way, where IASB1 gives some important additional insights. More specifically, it shows that (11) is the solution of a convex problem satisfying conditions (3$5)$, which proves that this per-user iterative update is nondecreasing and converges to a (univariate) local optimum, properties which were not known previously for DSB.

2) Iterative Approximation Spectrum Balancing 2 (IASB2): The per-user approximation of IASB1 can be further improved by the per-user approximation $f_{k}^{\mathrm{IASB} 2}$ of Table I. It adds an additional term to $f_{k}^{\mathrm{IASB} 1}$ with constant parameter $L$, which is used to make the approximation tighter in the interval $\mathcal{S}_{k}^{n}$ compared to that of IASB1. More specifically, a positive value for $L$ makes $f_{k}^{\mathrm{IASB} 2}$ a lower approximation of $f_{k}^{\mathrm{IASB} 1}$. Note that the value of $L$ cannot be chosen too large to ensure that $f_{k}^{\mathrm{IASB} 2}$ remains a convex overapproximation of $f_{k}$ in the interval $\mathcal{S}_{k}^{n}$. We propose the following positive value for $L$ :

$$
\begin{aligned}
L & =\min (x, y) \\
\text { with } \quad x & =\frac{w_{n}}{2\left(\hat{s}_{k}^{n}+\sum_{m \neq n} a_{k}^{n, m} \hat{s}_{k}^{m}+z_{k}^{n}\right)^{2}} \\
y & =\sum_{m \neq n} \frac{w_{m} \hat{s}_{k}^{m}\left(a_{k}^{m, n}\right)^{2}\left(\hat{s}_{k}^{m}+2 \sum_{p \neq m} a_{k}^{m, p} \hat{s}_{k}^{p}+2 z_{k}^{m}\right) / 2}{\left(\left(\hat{s}_{k}^{m}+\sum_{p \neq m} a_{k}^{m, p} \hat{s}_{k}^{p}+z_{k}^{m}\right)\left(\sum_{p \neq m} a_{k}^{m, p} \hat{s}_{k}^{p}+z_{k}^{m}\right)\right)^{2}},
\end{aligned}
$$

with $\hat{\mathbf{s}}_{k}=\left(s_{k}^{n \text {,mask }}, \tilde{s}_{k}^{-n}\right)$. The derivation of the proposed value for $L$ is omitted for space reasons, but it can be easily shown that this value guarantees that $f_{k}^{\text {IASB2 }}$ remains a convex upper bound to $f_{k}$ in the interval $\mathcal{S}_{k}^{n}$ and satisfies convergence conditions (3-5) at $\tilde{s}_{k}^{n}$. The improved tightness is stated by the following lemma:

Lemma 4.2: Per-user approximation $f_{k}^{\mathrm{IASB} 2}$ is tighter than approximations $f_{k}^{\mathrm{IASB} 1}$ and $f_{k}^{\mathrm{CA}-\mathrm{DSB}}$.

Proof: Proof trivial since $f_{k} \leq f_{k}^{\mathrm{IASB} 2} \leq f_{k}^{\mathrm{IASB} 1}$.

For the solution of the subproblems in (9) with $f_{k}^{\text {app }}=$ $f_{k}^{\mathrm{IASB} 2}$, we start from the corresponding KKT stationarity condition (10) with $f_{k}^{\text {app }}=f_{k}^{\mathrm{IASB} 2}$, and we observe that it can be reformulated as a simple quadratic equation in $s_{k}^{n}$. Subproblems in (9) can then be solved in closed-form by computing the roots of this quadratic polynomial and taking the best of the roots after projection on the interval $\mathcal{S}_{k}^{n}$. More formally:

$$
s_{k}^{n, *}=\underset{x \in \mathcal{X}}{\operatorname{argmin}} f_{k}^{\mathrm{IASB} 2}\left(x ; s_{k}^{-n}, \tilde{\mathbf{s}}_{k}\right)+\lambda_{n} x,
$$

with the set of candidate optimal solutions $\mathcal{X}$ defined as

$$
\mathcal{X}=\left\{\left[a_{1}\right]_{0}^{s_{k}^{n, \text { mask }}},\left[a_{2}\right]_{0}^{s_{k}^{n, \text { mask }}}\right\},
$$

with $a_{1}$ and $a_{2}$ denoting the roots of the quadratic polynomial.

In summary, approximation $f_{k}^{\mathrm{IASB} 2}$ is tighter than $f_{k}^{\mathrm{IASB} 1}$, but requires a slightly higher computational cost to solve the corresponding approximate problem in closed-form.

3) Iterative Approximation Spectrum Balancing 3 (IASB3): Our next method corresponds to the per-user approximation $f_{k}^{\text {app }}=f_{k}^{\text {IASB3 }}$ of Table I, which is obtained by a first-order linear overapproximation of terms (C) and (D) of $f_{k}$. Function $f_{k}^{\text {IASB3 }}$ is nonconvex in $s_{k}^{n}$. Its improved tightness is stated in the following lemma: 
Lemma 4.3: Per-user approximation $f_{k}^{\text {IASB3 }}$ is tighter than approximation $f_{k}^{\mathrm{IASB} 1}$ and $f_{k}^{\mathrm{CA}-\mathrm{DSB}}$.

Proof: Proof trivial since it is easy to see that $f_{k} \leq$ $f_{k}^{\mathrm{IASB} 3} \leq f_{k}^{\mathrm{IASB} 1} \leq f_{k}^{\mathrm{CA}-\mathrm{DSB}}$.

For IASB3, we observe that the KKT stationarity condition (10) can be rewritten as a cubic equation in $s_{k}^{n}$. The subproblems in (9) can then be solved in closed-form by computing the roots of a cubic polynomial and checking those roots after projection on the interval $\mathcal{S}_{k}^{n}$ in addition to the boundary points. More formally:

$$
s_{k}^{n, *}=\underset{x \in \mathcal{X}}{\operatorname{argmin}} f_{k}^{\mathrm{IASB} 3}\left(x ; s_{k}^{-n}, \tilde{\mathbf{s}}_{k}\right)+\lambda_{n} x,
$$

with the set of candidate optimal soluitons $\mathcal{X}$ defined as

$$
\mathcal{X}=\left\{0, s_{k}^{n, \text { mask }},\left[b_{1}\right]_{0}^{s_{k}^{n, \text { mask }}},\left[b_{2}\right]_{0}^{s_{k}^{n, \text { mask }}},\left[b_{3}\right]_{0}^{s_{k}^{n, \text { mask }}}\right\},
$$

and with $b_{1}, b_{2}, b_{3}$ the roots of the cubic equation.

We would like to highlight here that the IASB3 method corresponds to the ASB method of [1] if $b_{k}^{n}\left(\tilde{\mathbf{s}}_{k}\right)$ and $c_{k}^{n}\left(s_{k}^{-n}, \tilde{\mathbf{s}}_{k}\right)$ are fixed to zero. The addition of $b_{k}^{n}\left(\tilde{\mathbf{s}}_{k}\right)$ and $c_{k}^{n}\left(s_{k}^{-n}, \tilde{\mathbf{s}}_{k}\right)$ however ensures that IASB3 converges to a per-user local optimum, as opposed to ASB. One huge advantage of IASB3 compared to CA-DSB, SCALE, IASB1 and IASB2 is that the nonconvex nature of $f_{k}^{\text {IASB3 }}$ allows to get out of bad locally optimal solutions, as will be demonstrated in Section VI, which results in improvements in the data rate performance.

Finally we would like to highlight that a good choice of $q$ is relevant and determines the solution quality and convergence speed. The 'reference line' heuristics for ASB provided in [1], [3] can be used for this. A further analysis of this choice is a subject of further study.

4) Iterative Approximation Spectrum Balancing 4 (IASB4): A fourth method uses the approximation $f_{k}^{\text {IASB4 }}$ of Table I which is obtained by a first-order linear overapproximation of terms (C) and (D), and a nonlinear overapproximation of term (B) based on the following inequality (in the variable $v$ )

$$
-\log (1+v) \leq-\alpha_{k}^{q}\left(\tilde{\mathbf{s}}_{k}\right) \log (v)+\text { constant, }
$$

with the constants $\alpha_{k}^{q}\left(\tilde{\mathbf{s}}_{k}\right)=y /(1+y)$ and $y=$ $\tilde{s}_{k}^{q} /\left(\sum_{m \neq q} a_{k}^{q, m} \tilde{s}_{k}^{m}+z_{k}^{q}\right)$ chosen so that conditions (3-5) are satisfied for $f_{k}^{\text {IASB4 }}$ in $\tilde{\mathbf{s}}_{k}$. The overapproximation of term (B) is similar to that used by $f_{k}^{\mathrm{SCALE}}$, but then only applied in one term instead of all terms. Approximation $f_{k}^{\text {IASB4 }}$ is less tight compared to $f_{k}^{\mathrm{IASB} 3}$, because term (B) is replaced by an overapproximation. However, the advantage of $f_{k}^{\text {IASB}} 4$ is that the KKT stationarity condition (10) can be rewritten as a quadratic equation. Thus the subproblems in (9) can be solved in closed-form by computing the roots of a quadratic polynomial, instead of a cubic polynomial. Formally:

$$
s_{k}^{n, *}=\underset{x \in \mathcal{X}}{\operatorname{argmin}} f_{k}^{\mathrm{IASB} 4}\left(x ; s_{k}^{-n}, \tilde{\mathbf{s}}_{k}\right)+\lambda_{n} x,
$$

with the set of candidate optimal solutions $\mathcal{X}$ defined as

$$
\mathcal{X}=\left\{0, s_{k}^{n, \text { mask }},\left[c_{1}\right]_{0}^{s_{k}^{n, \text { mask }}},\left[c_{2}\right]_{0}^{s_{k}^{n, \text { mask }}}\right\},
$$

with $c_{1}$ and $c_{2}$ denoting the roots of the quadratic polynomial.

Similarly to IASB3, the IASB4 method corresponds to an approximation which is not necessarily convex in $s_{k}^{n}$. This allows to escape from a bad locally optimal solution when choosing a proper value for $q$, as will be shown in Section VI.

5) Iterative Approximation Spectrum Balancing 5 (IASB5): Our last proposed method uses the approximation $f_{k}^{\text {IASB5 }}$ of Table I which is obtained by a first-order linear overapproximation of term (D) of $f_{k}$ and a first-order nonlinear approximation of the form (15) for terms (B) and (C). For this nonconvex approximation, we observe that the KKT stationarity condition (10) can be rewritten as a cubic equation. Thus, the subproblems in (9) can be solved in closed-form by computing the roots of a cubic polynomial as follows:

$$
s_{k}^{n, *}=\underset{x \in \mathcal{X}}{\operatorname{argmin}} f_{k}^{\mathrm{IASB} 5}\left(x ; s_{k}^{-n}, \tilde{\mathbf{s}}_{k}\right)+\lambda_{n} x,
$$

with the set of candidate optimal solutions $\mathcal{X}$ defined as

$$
\mathcal{X}=\left\{0, s_{k}^{n, \text { mask }},\left[d_{1}\right]_{0}^{s_{k}^{n, \text { mask }}},\left[d_{2}\right]_{0}^{s_{k}^{n, \text { mask }}},\left[d_{3}\right]_{0}^{s_{k}^{n, \text { mask }}}\right\},
$$

with $d_{1}, d_{2}$ and $d_{3}$ the roots of the cubic equation.

Similarly to IASB3 and IASB4, the IASB5 method involves an approximation which is not necessarily convex in $s_{k}^{n}$, allowing to tackle the issue of getting stuck in bad locally optimal solutions when choosing good values for $q$ and $t$.

\section{ITERATIVE Fixed Point Update ImPlementation}

The CA-DSB and SCALE algorithms resort to iterative fixed point updates to solve their per-user approximations, instead of solving a polynomial of degree $N$. Similar iterative fixed point updates can be derived for our proposed methods. Similarly as in [2], [5], this can be done by starting from (10) and isolating one occurrence of $s_{k}^{n}$ to the left side of the equation sign to obtain a fixed point update form $s_{k}^{n}=\left[h\left(s_{k}^{n} ; s_{k}^{-n}, \tilde{\mathbf{s}}_{k}\right)\right]_{0}^{s_{k}^{n, \text { mask }}}$, with $h$ denoting the right side function in $s_{k}^{n}$, and with projection on $\mathcal{S}_{k}^{n}$. For IASB1, this fixed point update corresponds to (11). The drawbacks of these fixed point updates compared to the closed-form approach are (i) that one does not know how many updates are required to converge, (ii) that convergence to a local optimum of the per-user approximation is not guaranteed, and (iii) that the fixed point update approach for IASB3, IASB4 and IASB5 loses the beneficial ability to get out of bad locally optimal solutions. Performance of the fixed point update approach for our proposed methods will also be assessed in the next section.

\section{Vi. Simulations}

We consider 24AWG twisted pair lines. SNR gap is 12.9 $\mathrm{dB}$, corresponding to a coding gain of $3 \mathrm{~dB}$, a noise margin of $6 \mathrm{~dB}$ and a target symbol error probability of $10^{-7}$. Tone spacing $\Delta f$ is $4.3125 \mathrm{kHz}$. DMT symbol rate is $4 \mathrm{kHz}$.

\section{A. Improved Convergence}

The first scenario is a 7-user downstream ADSL scenario with line lengths $5 \mathrm{~km}, 4 \mathrm{~km}, 3.5 \mathrm{~km}, 3 \mathrm{~km}, 3 \mathrm{~km}, 2.5 \mathrm{~km}$, and $3 \mathrm{~km}$. The distances between central office and remote terminals are $0 \mathrm{~km}, 0 \mathrm{~km}, 0.5 \mathrm{~km}, 0.5 \mathrm{~km}, 3 \mathrm{~km}, 3 \mathrm{~km}$, and $3 \mathrm{~km}$, respectively. The maximum transmit power is $20.4 \mathrm{dBm}$. This scenario consists of 7 lines and 224 tones that reflect a whole range of different channel and interference settings. More specifically, it consists of lines with large and small 
signal-to-noise ratios, as well as large and small interferers. In total, it consists of $7 \times 224=1568$ different per-user per-tone problems, for which we compare the performance of the proposed iterative approximation methods. We started all methods from the same all-zero starting point. For this particular problem, it turned out that all methods converged to the same locally optimal solution (this is certainly not always the case as shown in Section VI-B), hence the comparison between numbers of approximations and iterations is fair.

The second colum of Table II shows the average number of per-user approximations to converge up to accuracy $0.1 \mathrm{dBm}$ of local optimum (using the proposed closed-form solution approach to solve the per-user approximations). It can be seen that the proposed methods require much fewer approximations to converge than CA-DSB and SCALE. In this case, IASB5 performs best, in particular requiring $69 \%$ fewer approximations than SCALE (for IASB5, we chose $\{q, t\}=\{6,5\}$ when $n \neq 5$ and $n \neq 6$, while $\{q, t\}=\{7,5\}$ and $\{q, t\}=\{6,7\}$ when $n=6$ and $n=5$, respectively).

The third column of Table II displays results when the fixed point update approach is used instead of the closed-form approach to solve the per-user approximations (up to accuracy $0.1 \mathrm{dBm})$. We can see that the proposed methods need two to three times fewer iterations to converge to a locally optimal solution compared to CA-DSB and SCALE.

\section{B. Improved Solution Quality}

The second scenario is a 6-user upstream VDSL scenario with line lengths $1.2 \mathrm{~km}, 1 \mathrm{~km}, 0.8 \mathrm{~km}, 0.6 \mathrm{~km}, 0.45 \mathrm{~km}$ and $0.3 \mathrm{~km}$. The maximum transmit power is $11.5 \mathrm{dBm}$. To demonstrate the improved data rate performance of the proposed methods, we plot the different per-user approximations for user 5 on tone 600 . The approximation point is chosen at the spectral mask $-30 \mathrm{dBm}$. It can be seen that the CA-DSB, SCALE, IASB1 and IASB2 approximations get stuck in a bad locally optimal solution at $-30 \mathrm{dBm}$ when solved using the closed-form approach, whereas the nonconvex approximations of IASB3, IASB4 and IASB5 succeed in getting out of the bad locally optimal solution and result in the true global optimum at $0 \mathrm{dBm}$. In fact, IASB3 matches the original nonconvex objective. Note however that the fixed point update approach gets stuck in $-30 \mathrm{dBm}$ for all methods.

Better behaviour of the nonconvex variants is also confirmed when looking at the final solution quality for the considered VDSL scenario: we saw an overall weighted data rate improvement of 3\% to 5\% for IASB3 and IASB5 compared to CA-DSB/SCALE, depending on the choice of $q$ and $t$.

\section{CONCLUSION}

Novel per-user iterative approximation methods have been proposed for DSL spectrum optimization. These methods involve improved convex as well as nonconvex per-user approximations that are much tighter and much simpler to solve (in closed-form) compared to existing ICA methods. The proposed methods therefore display a significantly improved convergence speed as much fewer approximations are required to obtain convergence, i.e., up to $69 \%$ reduction for the tested DSL scenarios. When using the iterative fixed point update implementation, the proposed methods require two to three
TABLE II

AVERAGE NUMBER OF APPROXIMATIONS AND FIXED POINT UPDATE ITERATIONS FOR DIFFERENT ITERATIVE APPROXIMATION METHODS

\begin{tabular}{|c|c|c|}
\hline Method & $\begin{array}{c}\text { Avg number of } \\
\text { per-user approximations }\end{array}$ & $\begin{array}{c}\text { Avg number of fixed } \\
\text { point update iterations }\end{array}$ \\
\hline CA-DSB & 3.147 & 4.195 \\
\hline SCALE & 5.557 & 6.385 \\
\hline IASB1 & 2.365 & 2.365 \\
\hline IASB2 & 2.291 & 2.291 \\
\hline IASB3 & 2.155 & 2.358 \\
\hline IASB4 & 2.155 & 2.358 \\
\hline IASB5 & $\mathbf{1 . 7 0 2}$ & $\mathbf{2 . 0 5 5}$ \\
\hline
\end{tabular}

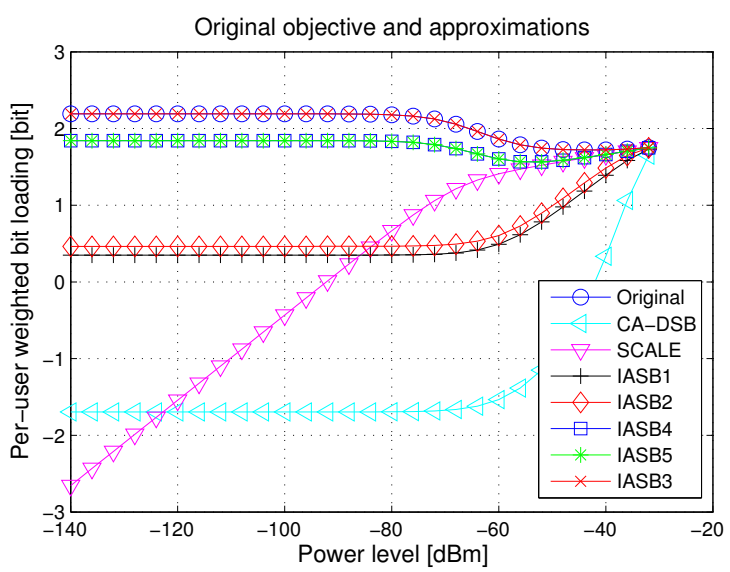

Fig. 1. Original nonconvex per-user objective and its approximations

times less iterations than CA-DSB or SCALE. Furthermore, it is shown that, when using closed-form solutions for per-user approximations, the nonconvex nature of the approximations used in IASB3, IASB4 and IASB5 allows to escape from bad locally optimal solutions, and can improve final solution quality (e.g., data rates) with respect to existing ICA methods.

\section{REFERENCES}

[1] R. Cendrillon, J. Huang, M. Chiang, and M. Moonen, "Autonomous spectrum balancing for digital subscriber lines," IEEE Transactions on Signal Processing, vol. 55, no. 8, pp. 4241-4257, Aug. 2007.

[2] P. Tsiaflakis, M. Diehl, and M. Moonen, "Distributed spectrum management algorithms for multiuser DSL networks," IEEE Transactions on Signal Processing, vol. 56, no. 10, pp. 4825-4843, Oct. 2008.

[3] C. Leung, S. Huberman, and T. Le-Ngoc, "Autonomous spectrum balancing using multiple reference lines for digital subscriber lines," in Proc. of GLOBECOM, Houston, TX, December 2010.

[4] W. Yu, "Multiuser water-filling in the presence of crosstalk," in Information Theory and Applications (ITA), San Diego, USA, Feb. 2007.

[5] J. Papandriopoulos and J. S. Evans, "Low-complexity distributed algorithms for spectrum balancing in multi-user DSL networks," in IEEE Int. Conf. on Communications, June 2006, vol. 7, pp. 3270-3275.

[6] R. B. Moraes and J. Rius i Riu B. Dortschy, A. Klautau, "Semiblind spectrum balancing for DSL," IEEE Transactions on Signal Processing, vol. 58, no. 7, pp. 3717-3727, Jul. 2010.

[7] Y. Xu, T. Le-Ngoc, and S. Panigrahi, "Global concave minimization for optimal spectrum balancing in multi-user DSL networks," IEEE Trans. on Signal Processing, vol. 56, no. 7, pp. pp. 2875-2885, Jul. 2008.

[8] P. Tsiaflakis, J. Vangorp, M. Moonen, and J. Verlinden, "A low complexity optimal spectrum balancing algorithm for digital subscriber lines," Signal Processing, vol. 87, no. 7, pp. 1735-1753, Jul. 2007.

[9] S. Huberman, C. Leung, and T. Le-Ngoc, "Dynamic spectrum management algorithms for multi-user xDSL," accepted for IEEE Communications Surveys and Talks, Sep. 2010.

[10] B.R. Marks and G.P. Wright, "A general inner approximation algorithm for nonconvex mathematical programs," Operations Research, vol. 26 , no. 4, pp. 681-683, 1978. 